\title{
Erythrosin B: a versatile colorimetric and fluorescent vital dye for bacteria
}

\author{
Josef D Franke*, , Ann L Braverman', Alison M Cunningham', Erin E Eberhard' \& Greg A Perry²
}

\section{ABSTRACT}

Rapidly assaying cell viability for diverse bacteria species is not always straightforward. In eukaryotes, cell viability is often determined using colorimetric dyes; however, such dyes have not been identified for bacteria. We screened different dyes and found that erythrosin B (EB), a visibly red dye with fluorescent properties, functions as a vital dye for many Gram-positive and -negative bacteria. EB worked at a similar concentration for all bacteria studied and incubations were as short as $5 \mathrm{~min}$. Given EB's spectral properties, diverse experimental approaches are possible to rapidly visualize and/or quantitate dead bacterial cells in a population. As the first broadly applicable colorimetric viability dye for bacteria, EB provides a cost-effective alternative for researchers in academia and industry.

\section{METHOD SUMMARY}

Incubation of erythrosin B with bacteria specifically stains membrane-compromised dead cells. Live and dead cells can then be quantified via bright-field microscopy or any number of instruments that quantitate fluorescence.

\section{KEYWORDS}

bacteria - cell viability - flow cytometry $\cdot$ membrane-exclusion dye - microscopy $\cdot$ vital dye

'Department of Biology, Creighton University, 2500 California Plaza, Omaha, NE 68178, USA; ${ }^{2}$ Department of Medical Microbiology \& Immunology, 2500 California Plaza, Omaha, NE 68178, USA; *Author for correspondence: jodyfranke@creighton.edu

BioTechniques 68: 7-13 (January 2020) 10.2144/btn-2019-0066
Determining cell viability is fundamental to many cell biological studies. Due to their ease of use, low cost and rapid results, numerous dyes are commonly used for cellviability determination in eukaryotes. Membrane-exclusion dyes (e.g., trypan blue and propidium iodide) are rapid and enter cells with compromised plasma membranes, such that dye-positive cells are scored as dead [1,2]. Other dyes, such as fluorescein diacetate (FDA) or methylene blue, indicate a cell's viability based on a particular metabolic activity $[3,4]$. For these dyes, the initially incubated dye is chemically altered by cellular enzymes. This results in a modified dye with new spectral properties that indicates living cells with metabolic activity. This diversity of eukaryotic dyes allows for cell viability to be determined based on the characteristics of either living cells or dead cells, and using either colorimetric or fluorescence-based methods of detection.

Unlike eukaryotes, no rapid colorimetric vital dyes are routinely used to assay cell viability in bacteria. Propidium iodide is often used as a vital dye in combination with other dyes that stain all cells (e.g., SYTO9), forming the basis of different live/dead assays such as the widely used BacLight ${ }^{\mathrm{TM}}$ kit [5]. There are downsides to the BacLight kits, in that they are costly and require expensive fluorescence quantitation equipment. Also, in these assays cells do not always fall into discrete live or dead categories as intermediate populations are possible [6,7]. Depending on the bacterial species being investigated, the concentration of each dye and the relative ratio of the two dyes often require optimization $[8,9]$. As a result, these kits are not well suited for field work, research involving multiple species or adherent cells in biofilms [10,11].

Alternatively, bacterial cell viability can be determined by the colony-forming units (CFUs) method [4]. Here, dilute solutions of cells are spread on agar plates and the number of colonies are counted after an incubation period and compared to control plates. This method has two significant drawbacks. First, the proportion of dead cells in a population can be overestimated because the method requires that all viable cells undergo sufficient rounds of division to form a visible colony. Living cells unable to divide, or dividing slower than controls, will be incorrectly scored as dead if they fail to divide sufficiently and form a colony in the appropriate amount of time. Second, the CFU method is particularly time consuming when working with slow-growing bacteria in which colony formation takes several days, resulting in undesirable waiting periods between experiments. To date, there are no colorimetric bacterial vital dyes available to researchers. Such a dye would save time and allow bacterial viability to be determined by light microscopy. It could also be used in experiments ranging from routine viability assays on a specific bacterial species to high-throughput screens identifying novel bactericidal compounds and analyzing multi-species samples.

Here, we show that erythrosin B (EB), a dye with colorimetric and fluorescent properties, functions as an indicator of bacterial viability. EB staining is rapid and works at a single concentration for diverse species of bacteria. The dye is inexpensive and allows for live/dead determination in both colorimetric and fluorescence-based assays for low, medium and high-throughput experimentation.

\section{METHODS \& MATERIALS}

\section{Organisms \& reagents}

The following bacteria were used in this study: Bacillus cereus (Carolina Biological, NC, USA 154870), Escherichia coli (MG1655), Klebsiella pneumoniae (Carolina Biological, 155095A), Pseudomonas fluorescencs (Carolina Biological, 155255), Serettia marcesans (Carolina Biological, 155450), Streptomyces albus (Ward's Science, NY, 


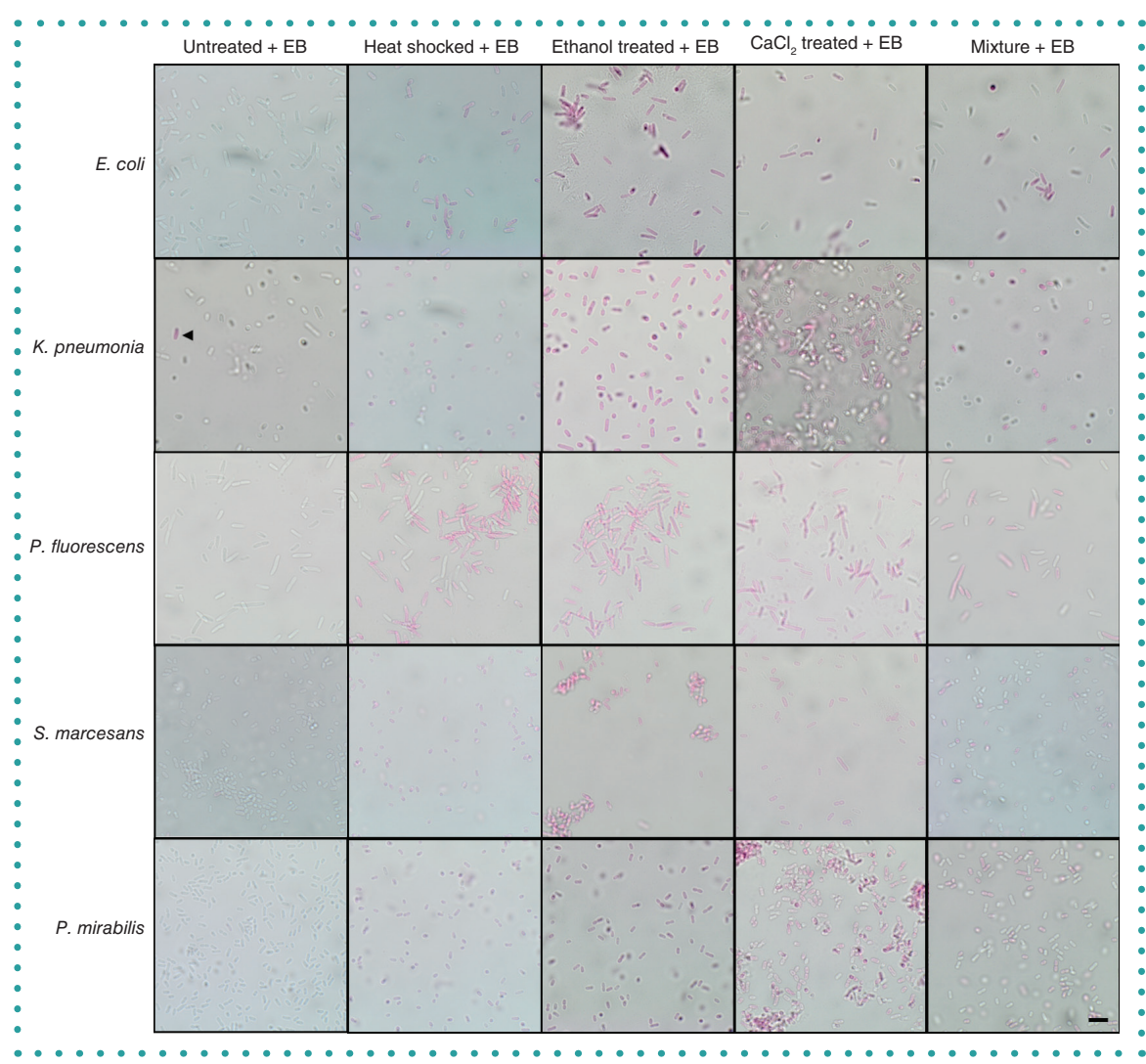

Figure 1. Erythrosin $B$ functions as a vital dye specifically staining dead or membrane-compromised Gram-negative bacteria. Bacterial cells were incubated with EB after heat shock, ethanol treatment or $\mathrm{CaCl}_{2}$ incubation. Images in the 'Mixture' column were taken of cell suspensions containing equal amounts of live, untreated cells and dead cells (either heat shocked or ethanol treated). Mixtures were incubated with EB and images show the ability to distinguish between living (dye-negative) and dead (dye-positive) cells. An arrowhead in the untreated control image (Klebsiella pneumoniae) indicates a clearly distinguishable dye-positive dead cell among dye-negative cells, further demonstrating the visible difference in staining between dye-positive and dye-negative cells. The scale bar in the bottom right panel is $5 \mu \mathrm{m}$ and applies to all panels. EB: Erythrosin B.

USA, 470179-180), Streptococcus mutans (Ward's Science, 470179-582), Proteus mirabilis (Ward's Science, 470179-138), Staphylococcus aureus (Carolina Biological, 155554) and Enterococcus faecalis (Ward's Science, 470179-184).

EB (Sigma, MO, USA, 200964-5G) was prepared by dissolving powder in $10 \mathrm{mM}$ Tris $\mathrm{pH}$ 7.5. Published values of the solubility of $\mathrm{EB}$ in aqueous solutions vary and may result from different preparations of the dye and from which supplier it is purchased. A small amount of undissolved dye was present when preparing a $0.1 \%(\mathrm{w} / \mathrm{v})$ solution. This stock solution was filtered through a $0.22-\mu \mathrm{m}$ filter to remove undissolved dye. To determine the concentration of this stock solution, a fresh dilute EB solution was prepared in which all dye fully dissolved after $10 \mathrm{~min}$ of stirring. This dilute solution was serially diluted and $A_{530}$ readings (the wavelength of maximal absorbance for EB) were collected, generating a reference relating $\mathrm{EB}$ dye concentration to $A_{530}$. The filtered stock solution of EB was then serially diluted, and absorbance values of the dilutions were compared to the reference absorbance values for the dilute solution. The stock EB concentration was determined to be $0.08 \%$ and is used throughout this work.

\section{Growth \& treatments of bacteria}

To prepare cells for viability studies, $3-\mathrm{ml}$ overnight bacterial cultures were grown in Luria broth. Fresh 5-ml cultures were inoculated from the overnight cultures and grown for 1-4 $\mathrm{h}$ until reaching an $\mathrm{OD}_{600}$ of $0.4-1.1$. Cells were harvested by centrifugation (15,000xg for $4 \mathrm{~min}$ ) and then resuspended in phosphate-buffered saline (PBS). A portion of these cells were kept at room temperature (untreated controls) while the remainder were transferred into separate microfuge tubes for different treatments. For heat shock treatment, microfuge tubes were placed at $70^{\circ} \mathrm{C}$ for 30 min before returning to room temperature. For ethanol treatment, $100 \%$ ethanol (Decon Labs, Inc. PA, USA, 2716) was added to cell suspensions to a final concentration of $30 \%$. Cell mixtures were incubated at $37^{\circ} \mathrm{C}$ for $30 \mathrm{~min}$ centrifuged, and then the pellet was resuspended in PBS. For $\mathrm{CaCl}_{2}$ treatment, cells were centrifuged and the pellet was resuspended in $1 \mathrm{ml}$ of ice-cold $100 \mathrm{mM} \mathrm{CaCl}_{2}$ and left on ice for $45 \mathrm{~min}$. After this initial incubation, the cells were centrifuged and resuspended in $100 \mu \mathrm{l}$ of $100 \mathrm{mM} \mathrm{CaCl}_{2}$.

\section{Assaying bacterial viability}

For bright-field microscopy assays, $10 \mu \mathrm{l}$ of each cell suspension (untreated, heat shocked, ethanol treated and $\mathrm{CaCl}_{2}$ treated) was mixed with $10 \mu \mathrm{l}$ of $0.8 \% \mathrm{~EB}$ and incubated for $5 \mathrm{~min}$ at room temperature. For $\mathrm{CaCl}_{2}$-treated cells, mixtures were placed at $42^{\circ} \mathrm{C}$ for $60 \mathrm{~s}$ before returning to room temperature for the remainder of the incubation. A total of $5 \mu$ l of the incubated dye:cell mixtures were taken to prepare wet mounts. Imaging was performed on a Zeiss Olympus BX61 microscope using an UPlanSApo 100X/1.14 NA oil objective lens with an Olympus $\mathrm{SC} 10$ camera.

For plate reading assays, a total of four optical densities (ODs) of log-phase cells were collected and evenly split between two tubes. Cells were centrifuged as described above. One tube was resuspended in $1 \mathrm{ml}$ of PBS (live cells), and the other tube was incubated at $70^{\circ} \mathrm{C}$ for $30 \mathrm{~min}$ (dead cells) before being resuspended in $1 \mathrm{ml}$ of PBS. A total of $100-\mu$ l cell mixtures were then prepared in triplicate by mixing appropriate volumes of live and dead cell suspensions to generate the following: $100 \%$ live cells, $75 \%$ live cells $/ 25 \%$ dead cells, $50 \%$ live cells $/ 50 \%$ dead cells, $25 \%$ live cells $/ 75 \%$ dead cells and $100 \%$ dead cells. A total of $100 \mu \mathrm{l}$ of $0.8 \%$ EB was added to all cell mixtures and incubated for $5 \mathrm{~min}$. Control cell mixtures containing $100 \mu \mathrm{l}$ of live cells or dead cells were incubated with $100 \mu$ l of PBS instead of EB. All mixtures were centrifuged and the supernatant removed. The tubes were briefly spun again and any residual supernatant was removed. Cell pellets were resuspended in $100 \mu \mathrm{l} \mathrm{PBS}$, vortexed and $75 \mu \mathrm{l}$ of 
each mixture was transferred to a clear 96-well plate (Nunc ${ }^{\mathrm{TM}}, 80042 \mathrm{LE}$ ). Absorbance readings (530 $\left.\mathrm{nm} ; \mathrm{A}_{530}\right)$ were taken using a Synergy $\mathrm{H} 1$ hybrid plate reader. Control $\mathrm{A}_{530}$ values were subtracted from the experimental $A_{530}$ values prior to calculations.

For flow cytometry assays, different EB cell suspensions were incubated in the presence of EB for $5 \mathrm{~min}$ at room temperature, centrifuged, and the cell pellets were resuspended in $1 \mathrm{mI}$ PBS. To remove excess unbound $E B$, the cells were washed twice in $1 \mathrm{ml}$ PBS prior to analysis. For the sodium azide time course, a $5 \mathrm{ml} S$. marcesans culture was grown to an $\mathrm{OD}_{600}$ of $\sim 0.2$ in Luria Broth at $37^{\circ} \mathrm{C}$. Sodium azide was added to a final concentration of $0.07 \%$ and $0.5 \mathrm{ml}$ samples were taken at different times points. Each sample was then immediately centrifuged to pellet the bacteria and stained with EB as described above to prepare samples for flow cytometry. A YETI flow cytometer (Propel Labs, Inc., CO, USA) was used to analyze cells (excitation with a $561 \mathrm{~nm}$ laser/ emission collected using a 583/30 filter).

\section{RESULTS \& DISCUSSION}

In eukaryotes, EB is known to function as a membrane-exclusion vital dye such that dead cells with compromised plasma membranes are dye positive [12]. Despite certain advantages over other traditional

Figure 3. The relative amount of dead cells in a bacterial suspension can be determined using erythrosin B absorbance values. Mixtures containing different ratios of cells ( $0 \%$ dead $/ 100 \%$ live; $25 \%$ dead $/ 75 \%$ live; $50 \%$ dead $/ 50 \%$ live; $75 \%$ dead $/ 25 \%$ live; and $100 \%$ dead $/ 0 \%$ live) were prepared and then similarly incubated with EB. In these assays, dead cells were prepared by heat shock. All mixtures were prepared in triplicate, and unbound EB was removed after the incubation. (A) Equal amounts of all Proteus mirabilis cell mixtures and controls (cells not exposed to EB: -EB) were transferred into separate wells of a 96-well plate and imaged to show the colorimetric variation of these different mixtures. (B-D) Comparable experiments were done with Escherichia coli and Klebsiella pneumoniae, and $\mathrm{A}_{530}$ values were collected for all three organisms and their mixtures. Control values (from boxed wells) were subtracted from each experimental value. The means and standard deviations were calculated for each mixture condition and plotted. Linear trends were observed for all species with $\mathrm{R}^{2}$ values as follows: E. coli (0.992), P. mirabilis (0.987) and $K$. pneumoniae (0.997).

EB: Erythrosin B.

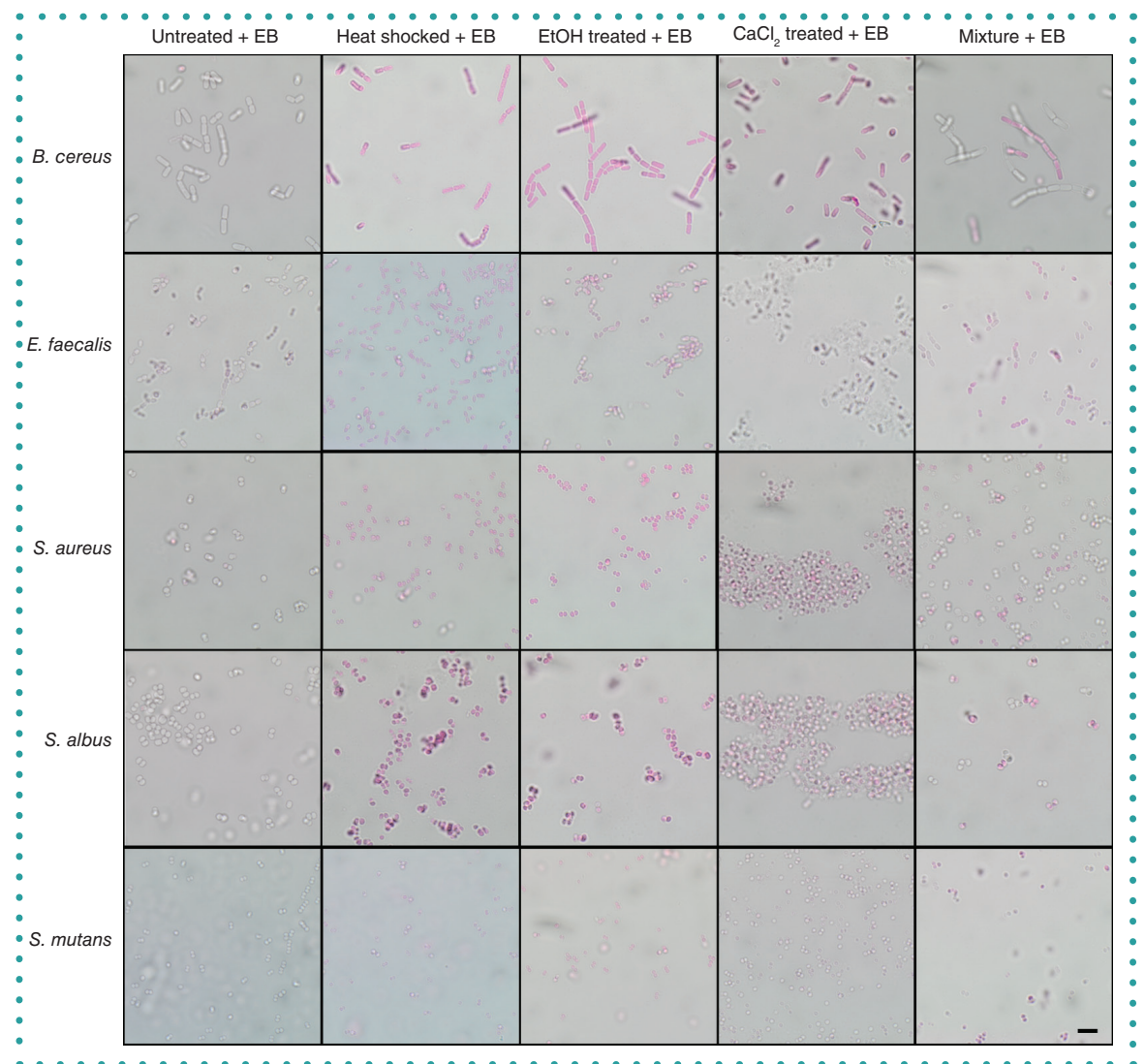

Figure 2. Erythrosin $B$ functions as a vital dye specifically staining dead or membrane-compromised Gram-positive bacteria. Bacterial cells were incubated with EB after heat shock, ethanol treatment or $\mathrm{CaCl}_{2}$ incubation. Images in the 'Mixture' column were taken of cell suspensions containing equal amounts of live, untreated cells and dead cells (either heat shocked or ethanol treated). Mixtures were incubated with EB and images show the ability to distinguish between living (dye-negative) and dead (dye-positive) cells. The scale bar in the bottom right panel is $5 \mu \mathrm{m}$ and applies to all panels. EB: Erythrosin B.

(A)

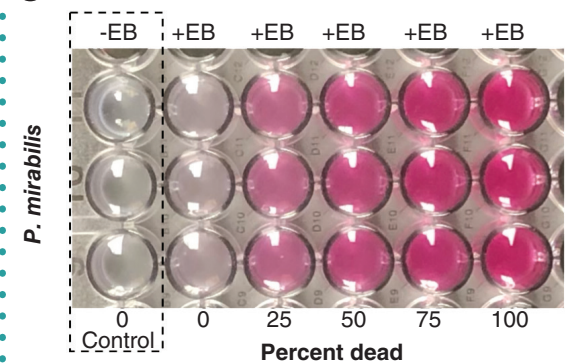

(c)

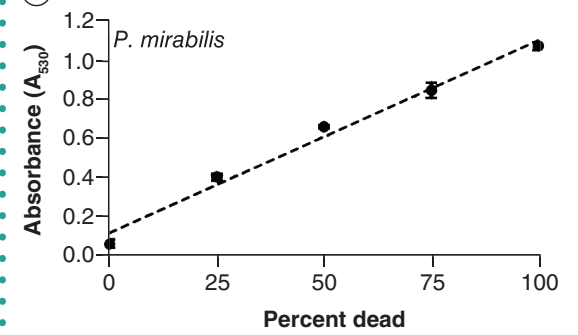

(B)

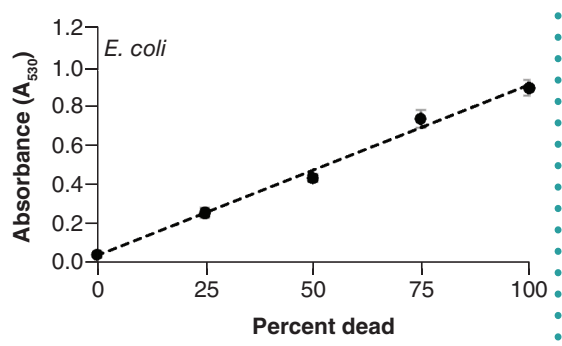

(D)

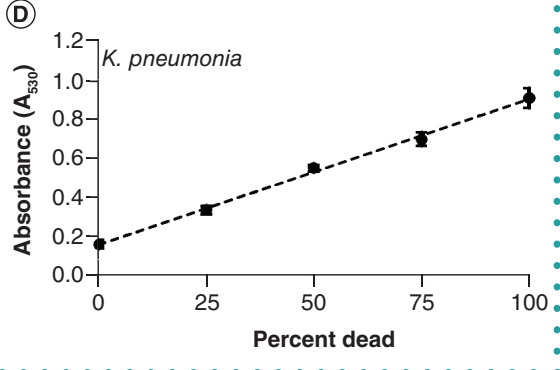



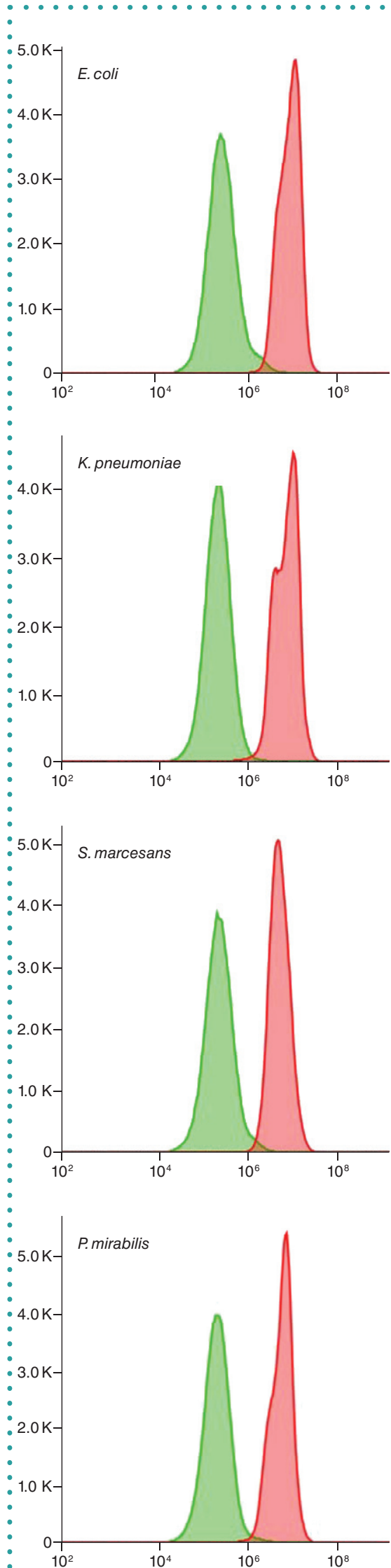
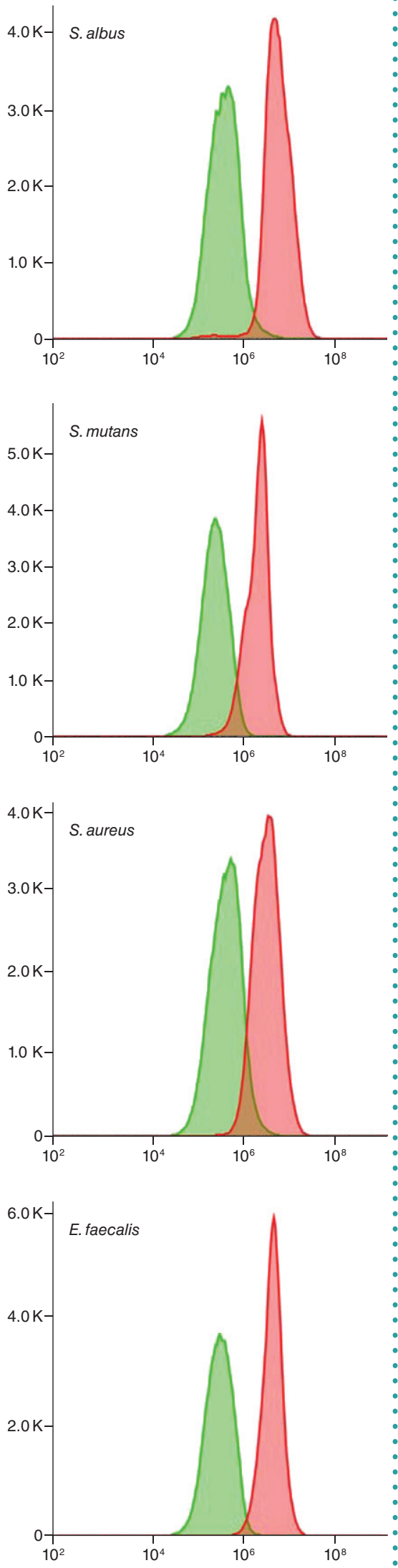

Figure 4. Distinguishing between live cell populations and dead cell populations via erythrosin B fluorescence. Clear differences in erythrosin B fluorescence intensity were observed when live (green plots) and dead cells (red plots) were analyzed by flow cytometry. In these assays, dead cells were prepared by ethanol exposure. Both Gram-negative bacteria (left panels) and Gram-positive bacteria (right panels) exhibited these differences. All flow cytometry plots indicate the number of cells analyzed on the $y$-axis and the fluorescence intensity (em. 583/30; $\log _{10}$ scale) on the x-axis. A minimum of 100,000 cells were analyzed for each species under each condition. 


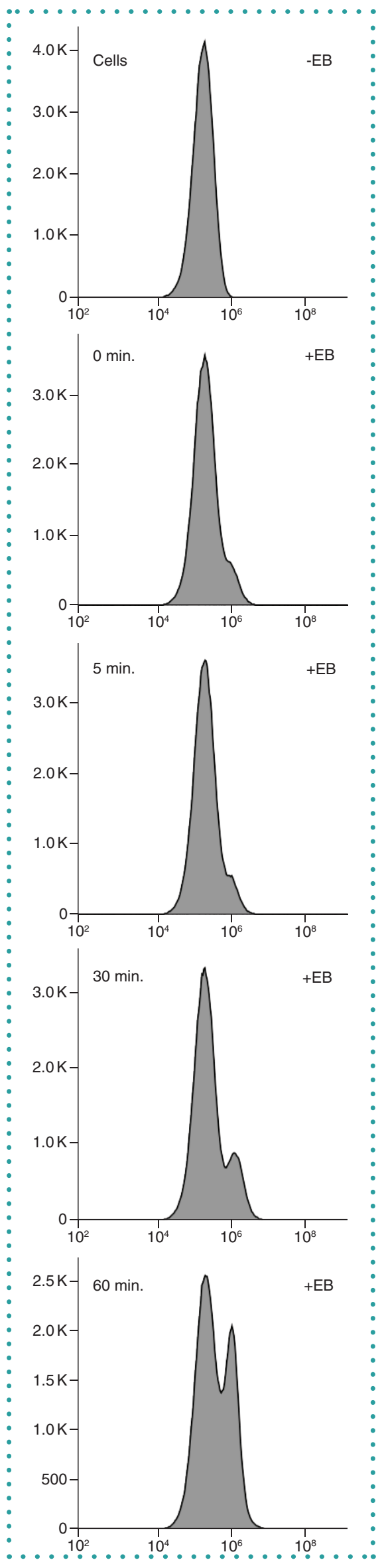

Figure 5. Monitoring cell viability in cultures exposed to a toxin. A Serettia marcesans culture was incubated with sodium azide and assayed for cell viability using EB at different time points. Prior to sodium azide addition, two samples were taken from the culture and analyzed directly. One sample was not incubated with EB (top panel) while the other one was incubated with the dye (0 min panel). At the indicated time points, samples were taken from the culture, incubated with EB and prepared for flow cytometry analysis. Over time, the relative amount of dye-positive cells in the culture increased. All flow cytometry plots indicate the number of cells analyzed on the $y$-axis and the fluorescence intensity on the $x$-axis. 100,000 cells were analyzed for each plot.

EB: Erythrosin B. colorimetric membrane-exclusion dyes [13], the application of EB as a vital dye in eukaryotes is not as widespread as other dyes with a comparable mechanism of action (e.g., trypan blue). We were searching for a practical vital dye for bacteria and tested EB due to its intense red color and spectral properties. The intense color would allow for a clear distinction between live and dead cells by bright-field microscopy for small cells like bacteria, and the spectral properties would allow a single dye to be used for cell-viability assays measuring either absorbance or fluorescence.

A variety of differently shaped Gramnegative and -positive bacteria were screened. Three different treatments were performed and designed to induce cell death (heat shock and ethanol exposure) or affect membrane permeability $\left(\mathrm{CaCl}_{2}\right.$ exposure) across diverse bacterial species. For all species examined in this study, EB specifically stained dead, or membranecompromised, Gram-negative (Figure 1) and -positive bacteria (Figure 2). For heatshocked and ethanol-treated organisms, virtually all treated cells were dye positive. For $\mathrm{CaCl}_{2}$-treated cells, not all treated cells were dye positive. $\mathrm{CaCl}_{2}$ treatment was designed to affect membrane permeability and not cause cell death, and resulted in a variable amount of dye-positive cells for different species. This variability likely reflects the inherent species-specific effects of $\mathrm{CaCl}_{2}$ exposure. Increased $\mathrm{CaCl}_{2}$ concentration, incubation time and/or prolonged heat shock would likely have resulted in a greater proportion of dye-positive cells.

For all species examined in this study, $<3 \%$ of healthy cells were dye positive by bright-field microscopy when incubated with EB (untreated columns in Figures 1 \& 2), demonstrating that EB does not readily stain living bacterial cells. EB-containing cell suspensions were generally viewed after 10-15 min of dye incubation, although clear differences between dye-negative and dye-positive cells were visible within $5 \mathrm{~min}$. Longer incubations, up to $30 \mathrm{~min}$, did not affect the ability to distinguish dye-positive from dye-negative cells. Faint staining of live bacteria was sometimes observed in three species (S. albus, S. mutans and $E$. faecalis) after typical incubations. However, the amount of staining in dye-positive dead cells was clearly increased compared 
with live cells, making it easy to distinguish between living and dead cells. Importantly, EB can also spatially resolve heterogeneities in the viability of different compartments in filamentous bacteria (Supplementary Figure 1), similar to the BacLight ${ }^{\text {TM }}$ system [9]. Lastly, visualization by bright-field microscopy allows for a rapid and accurate quantitation of dead cells in a sample with a low incidence of dead cells.

We examined the ability of EB to function as a general bacterial cell-viability indicator in different high-throughput assays based on its colorimetric and fluorescent properties. Mixtures of different ratios of living and dead cells were prepared for a variety of bacteria. These mixtures were incubated with EB for $5 \mathrm{~min}$, followed by the removal of unbound dye and the transfer of cells to a 96-well plate. $A_{530}$ measurements were collected and plotted for these different bacteria, with each exhibiting a linear trend based on EB absorbance (Figure 3). EB also functioned as a fluorescent bacterial cell-viability indicator by clearly distinguishing between living and dead cells for diverse bacterial species using flow cytometry (Figure 4). The extent of the fluorescence shift between live cells and dead cells did vary depending on the bacterial species (e.g., compare E. coli to $S$. aureus). These differences are likely due to either the relative cell size (larger cells will bind more dye) or inherent properties in the way in which EB binds to cellular materials. Together, these results demonstrate that it is possible to rapidly estimate the amount of dead cells in a population based on EB absorbance (Figure 3) and/or fluorescence (Figure 4) in different high-throughput assays.

To examine the ability of EB to assay changing cell viabilities in a bacterial culture, we treated an S. marcesans culture with a known toxin to Gram-negative bacteria sodium azide (Figure 5). Samples taken from the treated culture and examined by flow cytometry showed an increase in the amount of EB-positive cells over time. These results show that EB can detect changing cell viabilities rapidly and potentially be used to assay cell viability when screening possible antimicrobial agents.

For completeness, our research group primarily studies members of the Planctomycetes phylum, a diverse group of bacteria with evolutionarily unusual cell biological features (reviewed in [14]). In heat shock trials, EB functioned as vital dye for some, but not all Planctomycetes. In the exceptions, $20-30 \%$ of healthy cells were dye positive at the concentration used in this study ( $>90 \%$ of dead cells were dye positive), ruling this concentration of EB out as a viability indicator. It is possible that lower EB concentrations will work as a viability dye, or that some of these organisms have cell biological features making live cells more permeable to the dye.

All cell-viability assays have limitations. The CFU method can overestimate the proportion of dead cells because any viable cells that are unable to divide, or dividing more slowly than wild-type, will fail to form visible colonies and will be scored as dead. Membrane-exclusion dyes, such as EB and propidium iodine, can underestimate the proportion of dead cells, as recently dead cells may not have sufficiently compromised membranes to allow the dye to enter the cell. The sodium azide time course (Figure 5) suggests that EB stains cells soon after dying. How other viability methods score dying cells also varies, and it is often difficult to accurately quantitate or distinguish a dying cell population [4]. For membrane-exclusion dyes, a concern is whether the dye enters cells with mildly compromised membranes that may be dying but not dead. Chemical treatments that can mildly affect membrane permeability in bacteria (e.g., $20 \mathrm{mM}$ EDTA, $0.1-0.5 \%$ Triton $\mathrm{X} 100,0.12 \mathrm{M}$ guanidine $\mathrm{HCl}$ or $0.1 \%$ SDS) did not result in an observable increase in dye-positive live cells [unpublished observations]. The results suggest that $E B$ stains dead cells and not living cells with mildly compromised membranes.

EB is the first broadly applicable colorimetric vital dye for assaying viability in both Gram-positive and -negative bacteria. EB's spectral properties allow for its use in diverse experimental approaches with lowmedium- and high-throughput assays. This versatile bacterial vital dye could significantly reduce the cost and time associated with conducting viability studies on diverse bacterial species as well as experiments involving multiple species. Optimization of dye concentrations for different species is likely unnecessary, as this is an individual dye that works well at a single concentration.

\section{FUTURE PERSPECTIVE}

Unlike existing methods, EB's colorimetric properties allow for rapid, straightforward live/dead determination by bright-field microscopy with a single dye. Importantly, its sensitivity allows for low rates of death to be accurately quantified. EB has diverse commercial and academic applications such as studies screening for new antimicrobial compounds and determining the concentration of these needed for bactericidal effects. EB staining may be particularly useful for field studies looking at diverse microbiomes, in field hospitals in the developing world where equipment and resources are limited, and in studying or identifying pathogenic bacteria that have entered a viable but nonculturable state [15]. Last, while this study examined EB's utility as an individual dye, future studies may identify important uses for it in bacterial viability studies as part of dye mixtures.

\section{SUPPLEMENTARY DATA}

To view the supplementary data that accompany this paper please visit the journal website at: www.future-science. com/doi/suppl/10.2144/btn-2019-0066

\section{AUTHOR CONTRIBUTIONS}

JDF conceived and designed the experimental study, collected data and wrote the manuscript; ALB, AMC and EEE performed experiments and collected data; and GAP designed experiments and provided technical assistance.

\section{ACKNOWLEDGMENTS}

The authors would like to thank Mackenzie Taylor for use of her microscope for imaging and Charles Deutch for providing the bacterial strains used in this study. The authors would also like to thank Jill Blankenship, Charles Deutch and Soochin Cho for comments and suggestions on the manuscript. Creighton University has applied for a provisional patent in the United States for using this dye as a live/dead bacteria indicator.

\section{FINANCIAL \& COMPETING INTERESTS DISCLOSURE}

The authors have no relevant affiliations or financial involvement with any organization 
or entity with a financial interest in or financial conflict with the subject matter or materials discussed in the manuscript. This includes employment, consultancies, honoraria, stock ownership or options, expert testimony, grants or royalties.

No writing assistance was utilized in the production of this manuscript.

\section{OPEN ACCESS}

This work is licensed under the AttributionNonCommercial-NoDerivatives 4.0 Unported License. To view a copy of this license, visit http://creativecommons.org/licenses/ by-nc-nd/4.0/

\section{REFERENCES}

1. Crowley LC, Marfell BJ, Christensen ME, Waterhouse NJ. Measuring cell death by trypan blue uptake and light microscopy. Cold Spring Harb. Protoc. 2016(7), (2016).

2. Crowley LC, Scott AP, Marfell BJ, Boughaba JA Chojnowski G, Waterhouse NJ. Measuring cell death by propidium iodide uptake and flow cytometry. Cold Spring Harb. Protoc. 2016(7), (2016).

3. Altman SA, Randers L, Rao G. Comparison of trypan blue dye exclusion and fluorometric assays for mammalian cell viability determinations. Biotechnol. Prog. 9(6), 671-674 (1993)

4. Kwolek-Mirek M, Zadrag-Tecza R. Comparison of methods used for assessing the viability and vitality of yeast cells. FEMS Yeast Res. 14(7), 1068-1079 (2014).

5. Boulos L, Prevost M, Barbeau B, Coallier J, Desjardins R. LIVE/DEAD BacLight: application of a new rapid staining method for direct enumeration of viable and total bacteria in drinking water. J. Microbiol. Methods 37(1), 77-86 (1999).

6. Berney M, Hammes F, Bosshard F, Weilenmann HU, Egli T. Assessment and interpretation of bacterial viability by using the LIVE/DEAD BacLight Kit in combination with flow cytometry. Appl. Environ. Microbiol. 73(10) 3283-3290 (2007).

7. Virta $M$, Lineri S, Kankaanpaa $P$ et al. Determination of complement-mediated killing of bacteria by viability staining and bioluminescence. Appl. Environ. Microbiol.
64(2), 515-519 (1998)

8. Stiefel P, Schmidt-Emrich S, Maniura-Weber K, Ren Q Critical aspects of using bacterial cell viability assays with the fluorophores SYTO9 and propidium iodide. BMC Microbiol. 15(1), 36 (2015).

9. Stocks SM. Mechanism and use of the commercially available viability stain, BacLight. Cytometry A 61(2) 189-195 (2004).

10. Rosenberg M, Azevedo NF, Ivask A. Propidium iodide staining underestimates viability of adherent bacteria cells. Sci. Rep. 9(1), 6483 (2019)

11. Netuschil L, Auschill TM, Sculean A, Arweiler NB. Confusion over live/dead stainings for the detection of vital microorganisms in oral biofilms - which stain is suitable? BMC Oral Health 14, 2 (2014).

12. Bhuyan BK, Loughman BE, Fraser TJ, Day KJ. Comparison of different methods of determining cell viability 97(2), 275-280 (1976).

13. Krause AW, Carley WW, Webb WW. Fluorescent erythrosin $B$ is preferable to trypan blue as a vital exclusion dye for mammalian cells in monolayer culture. $J$. Histochem. Cytochem. 32(10), 1084-1090 (1984).

14. Wiegand S, Jogler M, Jogler C. On the maverick Planctomycetes. FEMS Microbiol. Rev. 42(6), 739-760 (2018).

15. Oliver JD. Recent findings on the viable but nonculturable state in pathogenic bacteria. FEMS Microbiol. Rev. $34(4), 415-425$ (2010). 